\title{
Visible AO Observations at Halpha for Accreting Young Planets
}

\author{
L.M. Close ${ }^{1}$, K. Follette ${ }^{1}$, J.R. Males ${ }^{1}$, K. Morzinski ${ }^{1}$, T.J. Rodigas ${ }^{1}$, \\ P. Hinz ${ }^{1}$, Y-L. Wu ${ }^{1}$, D. Apai ${ }^{1}$, J. Najita ${ }^{4}$, A. Puglisi ${ }^{2}$, S. Esposito ${ }^{2}$, \\ A. Riccardi ${ }^{2}$, V. Bailey ${ }^{1}$, M. Xompero ${ }^{2}$, R. Briguglio ${ }^{2}$ \\ and A. Weinberger ${ }^{3}$ \\ ${ }^{1}$ Steward Observatory, University of Arizona, Tucson, AZ 85721, USA \\ ${ }^{2}$ INAF - Osservatorio Astrofisico di Arcetri, I-50125, Firenze, Italy \\ ${ }^{3}$ Carnegie DTM, Washingtion DC. USA \\ ${ }^{4}$ NOAO, Tucson AZ 85721, USA
}

\begin{abstract}
We utilized the new high-order (250-378 mode) Magellan Adaptive Optics system (MagAO) to obtain very high-resolution science in the visible with MagAO's VisAO CCD camera. In the good-median seeing conditions of Magellan $(0.5-0.7 ")$ we find MagAO delivers individual short exposure images as good as 19 mas optical resolution. Due to telescope vibrations, long exposure $(60 \mathrm{~s})$ r' $(0.63 \mu \mathrm{m})$ images are slightly coarser at FWHM $=23-29$ mas (Strehl $\sim 28 \%$ ) with bright $(R<9 \mathrm{mag})$ guide stars. These are the highest resolution filled-aperture images published to date. Images of the young $(\sim 1 \mathrm{Myr})$ Orion Trapezium $\theta^{1}$ Ori A, B, and C cluster members were obtained with VisAO. In particular, the 32 mas binary $\theta^{1}$ Ori $C_{1} C_{2}$ was easily resolved in non-interferometric images for the first time. Relative positions of the bright trapezium binary stars were measured with $\sim 0.6-5$ mas accuracy. In the second commissioning run we were able to correct 378 modes and achieved good contrasts (Strehl> 20\% on young transition disks at $H \alpha$ ). We discuss the contrasts achieved at $H \alpha$ and the possibility of detecting low mass $(\sim 1-5$ Mjup) planets (past 5AU) with our new SAPPHIRES survey with MagAO at $H \alpha$.
\end{abstract}

Keywords. Extra-solar Planets, Adaptive Optics

\section{Introduction to Visible AO and the Magellan AO System}

To reach the maximum resolution (and use important recombination lines) with modern telescopes one must work blueward of the NIR in the visible. So called "visible AO" can theoretically reach 19 mas resolutions on an $6.5 \mathrm{~m}$ telescope at $0.656 \mu \mathrm{m}(\mathrm{H \alpha})$. We note that AO with "lucky" imaging in the visible has been successfully used at the somewhat smaller 5m Palomar (Law et al. 2009) and has reached resolutions of 35 mas. In the near future some polarization work will be done in the visible with the 8m VLT with the SPHERE AO system and ZIMPOL (Bazzon et al. 2012). Yet, MagAO is the first large $(D \geqslant 6.5 \mathrm{~m})$ telescope AO system designed to work in the visible -complete with a facility CCD AO science camera (VisAO). The MagAO commissioning results presented here inform us on the utility of large telescope visible AO performance.

We have developed an AO system (inspired, in large part, by LBT's FLAO system; Esposito et al. (2012)) that can reach 20 mas resolutions with just 250-378 modes at 1 $\mathrm{kHz}$ sampling speeds.

MagAO, with its VisAO camerat, is the first large telescope $(\geqslant 6.5 \mathrm{~m})$ facility AO system deployed that is targeting science in the visible $(0.6-1.1 \mu \mathrm{m})$. As is shown in

$\dagger$ see http://visao.as.arizona.edu/for web resources, SPIE publications, and guides for observers 


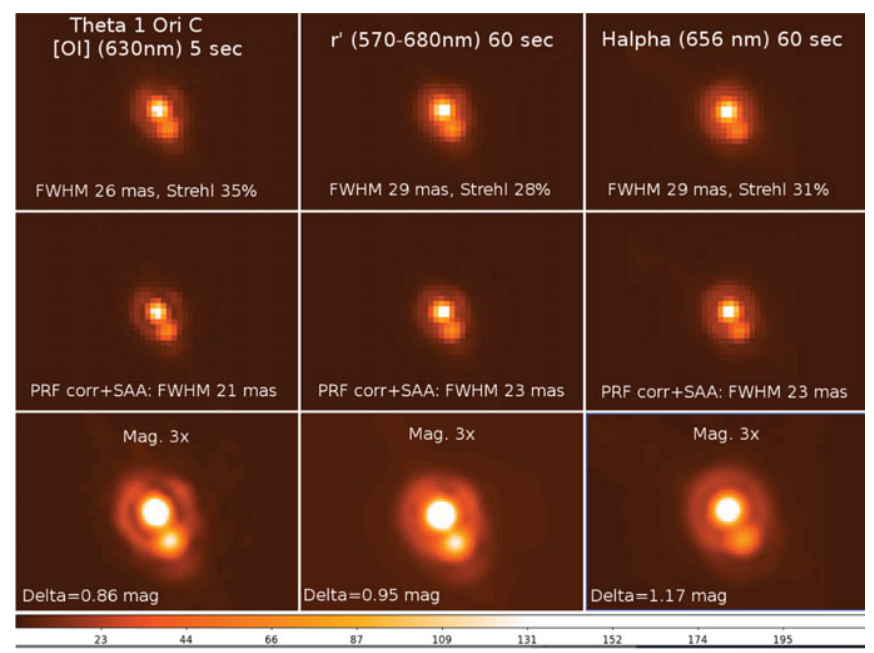

Figure 1. Top row: the central ionizing binary of the Trapezium: $\theta^{1}$ Ori $\mathrm{C}$ as imaged with MagAO's VisAO CCD camera in different filters. Note the excellent resolution in the raw 60 second image. We note that no post-detection shift and add (SAA) was applied, nor was there any frame selection used to produce these top row images. Typically we achieved resolutions of $0.026-0.029$ " and Strehls of $28-35 \%$ in $0.5-0.7$ " V-band seeing. Middle row: the same data as the top row, except the images have been post-detection aligned (SAA) and the pixel response function (PRF) has been removed. This improved image resolution by $\sim 5-6$ mas. Bottom row: the row above is magnified by $3 \times$ to better display the data of the middle row. These are the highest resolution, deep, images ever obtained to our knowledge. Figure reproduced from Close et al. (2013a).

figure 1, MagAO (at first light) produced long exposure (60s) diffraction-limited (110 nm WFE; $28 \%$ Strehl) $0.63 \mu \mathrm{m}$ images. For more technical details about MagAO itself please see Close et al. (2012a).

\section{Contrasts at $H \alpha$ and the SAPPHIRES $H \alpha$ Survey}

We estimate the contrasts achieved $(\Delta H \alpha=9.5 \mathrm{mag}$ at 70 mas $)$ at $H \alpha$ from almost 2 hours of open shutter observations of a young Tauri star. The data were reduced with an ADI reduction. See Close et al. (2013b) for more details of these contrasts.

We have started a Survey for Accreting Proto Planets at Halpha and in IR Emission in transitional diskS: SAPPHIRES. This will be a survey in both $H \alpha$ and simultaneously in the L' (3.8 microns) band. In deep 2 hour on-source images we expect to detect $\sim 7$ accreting planets in the gaps of 24 nearby transitional disks. Again please see Close et al. (2013b) for more details about SAPPHIRES.

\section{References}

Bazzon, Andreas et al. (2012) Proceedings of the SPIE, Volume 8446, id. 844693-844693-14

Close, L. M. et al. 2012a Proc. SPIE, Volume 8447, id. 84470X-84470X-16

Close, L. M. et al. 2012b ApJ, 749, article id. 180.

Close, L. M. et al. 2013 ApJ, 774, 94

Close, L. M. et al. 2013 ApJ Lett, submitted

Esposito, S. et al. 2012 proc. SPIE Volume 8447, article id. 84470U

Law, N. M.; Mackay, C. D.; Dekany, R. G.; Ireland, M.; Lloyd, J. P.; Moore, A. M.; Robertson, J. G.; Tuthill, P.; Woodruff, H. C. 2009, ApJ 692, 924. 